\title{
Avances y desafíos de la evaluación nacional de los rendimientos escolares en Guatemala
}

\section{Progress and challenges of national evaluation students' performance in Guatemala}

Bienvenido Argueta Hernández ${ }^{1}$

\section{Resumen}

Una de las expresiones de la globalización en el campo de la educación formal, ha sido la reducción de la calidad educativa a una expresión de los resultados del rendimiento escolar en las áreas de matemática y comprensión lectora, como códigos básicos del acceso al sistema económico neoliberal. En Guatemala, esta dinámica no ha sido la excepción, poniendo en riesgo elementos básicos de formación ciudadana en el marco de una sociedad multicultural y con una historia que reclama el reconocimiento de las diferencias. A pesar de los Acuerdos de Paz, el modelo globalizador se impuso frente a los esfuerzos del establecimiento de una educación pertinente y relevante a la sociedad guatemalteca, imponiendo parámetros de calidad que restringen la construcción de ciudadanías multiculturales. El presente artículo hace un balance y un examen de las políticas de la calidad y de la evaluación del rendimiento escolar en Guatemala.

Palabras clave: calidad educativa, evaluación, pruebas de rendimiento académico, ciudadanía, multiculturalidad

\begin{abstract}
One of the expressions of globalization in the field of formal education has been the reduction of educational quality to an expression of the results of school performance in the areas of
\end{abstract}

\footnotetext{
${ }^{1}$ Doctor en Educación, Director del Instituto de Investigaciones Educativas de la Escuela de Formación de Profesores de Enseñanza Media de la Universidad de San Carlos de Guatemala. ORCID 0000-0001-7121-2009/bienvenidoargueta@gmail.com Recibido 03 de septiembre de 2019 / Aceptado 04 de noviembre de 2019
} 
mathematics and reading comprehension as basic codes of access to the neoliberal economic system. In Guatemala, this dynamic has not been the exception, putting at risk basic elements of citizen formation within the framework of a multicultural society and with a history that demands the recognition of differences. Despite the Peace Agreements, the globalizing model was imposed against the efforts of establishing a relevant and relevant education to Guatemalan society, imposing quality parameters that restrict the construction of multicultural citizenships. This article takes stock and examines quality policies and the evaluation of school performance in Guatemala.

Keywords: educational quality, evaluation, academic performance test, citizenship, multiculturalism

\section{Introducción}

Desde las últimas dos décadas el concepto de calidad educativa ha estado asociado de manera sistemática con los indicadores de eficiencia interna del sistema educativo, transfiriendo un mayor peso hacia los resultados en las evaluaciones del rendimiento escolar en las áreas de lectura y matemáticas. De hecho, los esfuerzos llevados a cabo con las reformas educativas de mediados y finales de los años 80 del siglo XX, enfatizaban en la pertinencia y relevancia curricular con acciones de descentralización que promovían mayor participación del magisterio y las comunidades en los proyectos educativos a nivel de aula (Ministerio de Educación, MINEDUC, 1988).

En este sentido, la evaluación de los aprendizajes adquirió un enfoque de apoyo hacia la revisión y sustento de las innovaciones que tenían como foco central, la adecuación de la oferta educativa a las necesidades de la población. En términos concretos se promovieron dos acciones fundamentales que consistieron, en primer lugar, en la evaluación de los logros en idioma español y matemática de los alumnos de sexto grado de primaria en Guatemala, por parte del Instituto de Investigaciones Educativas de la Universidad de San Carlos de Guatemala, en coordinación con el Sistema Nacional de Mejoramiento de los Recursos Humanos y Adecuación Curricular (SIMAC), dependencia del MINEDUC en el año 1989 
(Chávez, 1989). La finalidad de esta evaluación consistía en recoger elementos básicos para revisar y retroalimentar las guías curriculares que fueron diseñadas en el inicio del proceso democrático posterior a los regímenes militares. En segundo lugar, en 1992 se fortaleció el Departamento de Investigación y Evaluación del SIMAC con la generación de un Centro Nacional de Pruebas, el cual llevó a cabo una evaluación para los tres primeros grados de la primaria en lectura y matemáticas, teniendo una muestra de lo urbano y lo rural.

\section{Discusión teórica}

A finales de los años 90, se observó el retorno a una centralización del currículo y un aumento a los mecanismos de control por la vía de las evaluaciones educativas. No obstante, las expectativas para profundizar las reformas dirigidas al reconocimiento y valoración de los pueblos indígenas crecieron con la firma de los Acuerdos de Paz, que ponían fin al conflicto armado interno de más de treinta años, en realidad se convirtieron en una nueva dinámica que por un lado intentaba la privatización de la educación, y por el otro una homogenización de la propuesta educativa. Estas circunstancias fueron determinadas por el curso posterior a la caída del muro de Berlín y las dinámicas globalizadoras en el ámbito educativo.

El Centro Nacional de Pruebas del SIMAC, fue disuelto en 1997 y se trasladó a la Universidad del Valle de Guatemala con el nombre de Programa Nacional de Evaluación del Rendimiento Escolar (PRONERE). Este proceso de cambio hacia una universidad privada fue una decisión del Ministerio de Educación con el apoyo del Banco Mundial. El PRONERE inició con un pilotaje de pruebas, y en 1998 llevó a cabo su primera evaluación para tercero y sexto grado del nivel primario y tercero básico del nivel medio (secundaria) en matemáticas y lectura. Uno de los elementos más innovadores en el marco de estas evaluaciones fue que para tercer grado del nivel primario, las mediciones se realizaron en los 4 idiomas mayas mayoritarios: K'iche', Kaqchikel, Q’eqchi’ y Mam, no obstante, constituían una traducción de las pruebas en castellano (MINEDUC, 1999). Los informes de las evaluaciones regularmente se manejaron de manera reservada y se solicitaba su acceso directamente al MINEDUC, cuestión que contrastaba con la posibilidad de que los investigadores pudieran publicar en 
revistas extranjeras. El proyecto se desarrolló hasta el año 2004 en la Universidad del Valle de Guatemala (Fortín, 2013). Las evaluaciones cobraron una influencia cada vez mayor a partir del año 2004, tomando como referencia la publicación del texto "Mañana es muy tarde", por parte del Programa de Promoción de la Reforma Educativa en América Latina y el Caribe (PREAL, 2000). Esta declaración ideológica repercutió en un concepto de calidad equivalente a los resultados en las evaluaciones de rendimiento escolar, y al igual que programas similares como el "No Child Left Behind", se transfería a los docentes la responsabilidad mayor. ${ }^{2}$ Sin embargo, a las reformas generadas a partir del año 2000 con el cambio del Currículo Nacional Básico, se incorporaron estándares que en realidad confundieron a los docentes que ya tenían dificultades para comprender las competencias y llevarlas a la práctica y no lograron elevar el rendimiento escolar en las diferentes evaluaciones.

En todo caso, la incorporación de los estándares en el Ministerio de Educación, con el apoyo de la Agencia Internacional de los Estados Unidos para el Desarrollo (USAID), señalaban esta ruta de acción definiendo un estándar educativo como:

Un indicador de medida de progreso hacia la calidad educativa... Las evaluaciones nacionales para las y los alumnos de tercero básico y graduandos son los instrumentos utilizados para monitorear el progreso de los estudiantes en cuanto al logro de los estándares educativos (MINEDUC, 2008, p.3).

Es importante señalar que la vinculación entre los resultados desfavorables de los estudiantes y la responsabilidad docente en cuanto a la pretensión de implicar los resultados en las evaluaciones para otorgar los aumentos salariales, también se inició una nueva dinámica de liderazgo dentro del MINEDUC. El nuevo discurso de calidad como equivalente a los resultados en las evaluaciones tuvo como fuente principal al sector empresarial y los principales tanques de pensamiento, quienes insistían en la incorporación de los estándares

\footnotetext{
2 Es un hecho universal que las políticas laborales, salariales y de incentivos suelen privilegiar la antigüedad en el servicio y la presentación de credenciales académicas, y no el desempeño en la labor docente. Esto hace muy difícil a los padres de familia y usuarios del servicio educativo retener maestros altamente calificados y sustituir a los maestros deficientes. Es preciso plantearse formas de incrementar las remuneraciones docentes en plazos específicos, pero acompañadas de reformas a los estatutos legales que permitan vincular estos incrementos al desempeño docente y a los logros en el rendimiento de los alumnos.
} 
y centrar el aprendizaje en aspectos directamente vinculados hacia la vida adulta y la productividad. A este respecto el documento de PREAL (2000), señala que cada país debe establecer estándares educativos claros, sistematizar las pruebas en el ámbito nacional y fijar medidas de evaluación del impacto de sus políticas educativas. La medición del aprendizaje de los alumnos suministrará un insumo importante para evaluar el desempeño de docentes y directores de escuela. Asimismo, los empresarios son quienes tienen mayor conciencia de cómo la calidad de la educación afecta la productividad y determina las oportunidades de competitividad.

Todos los esfuerzos para atender los compromisos de los Acuerdos de Paz, particularmente el Acuerdo Sobre Identidad y Derechos de los Pueblos Indígenas que mandaba una reforma educativa para la formación de una ciudadanía intercultural, prácticamente se disolvieron en términos de que la preocupación se convirtió en la occidentalización de los procesos de aprendizaje orientadas por las evaluaciones educativas. De esa cuenta, la educación bilingüe se ha reducido hasta llegar, en últimas instancias a ser una acción que se justifica si se garantiza mejores resultados en la lectura en castellano y en matemáticas, olvidando así su auténtica razón de ser. Adicionalmente que uno de los factores asociados a los rendimientos más bajos se encuentra en la adscripción étnica y lingüística en razón de las dinámicas de pobreza, discriminación y exclusión en que se encuentran los pueblos indígenas. ${ }^{3}$

En el año 2005, el Programa de Evaluación fue transferido al MINEDUC, creando primero el Sistema Nacional de Evaluación e Investigación Educativa (SINEIE), y en el 2008 se transformó en la Dirección General de Evaluación e Investigación Educativa (DIGEDUCA), con el apoyo y supervisión de USAID. Hasta el año 2010, se realizaron seis evaluaciones para los grados de tercero y sexto del nivel primario. En el caso de los estudiantes del tercer curso del ciclo básico del nivel medio, se llevaron a cabo cuatro durante las últimas décadas, siendo la última en el año 2013. Recientemente, se amplían las evaluaciones de los graduandos

\footnotetext{
${ }^{3}$ Véase: Ministerio de Educación (2010) La repitencia en primer grado, factores que influyen e impacto en los grados siguientes. Dirección General de Evaluación e Investigación Educativa, Guatemala; Ministerio de Educación (2017) Informe de Factores Asociados al rendimiento de los estudiantes en la evaluación de primaria, Dirección General de Evaluación e Investigación Educativa, Guatemala.
} 
(último año del ciclo diversificado del nivel medio o secundaria), siempre en las áreas de lenguaje y matemáticas. En efecto, desde hace 14 años se han realizado las evaluaciones año tras año a los graduandos.

Además de la evaluación de los aprendizajes de los estudiantes, la DIGEDUCA ha realizado investigaciones variadas que van desde diagnósticos de violencia escolar, factores asociados al rendimiento escolar y otros estudios con fondos del Ministerio de Educación o de agencias de cooperación internacional. Pero también se ha evaluado a maestros como parte del requisito para el ingreso al proceso de la contratación docente.

Por otra parte, la evaluación en Guatemala se ha incorporado a la dinámica regional y mundial. Las evaluaciones que desde 1989 tenían como marco referencial el orden nacional por excelencia, recientemente se han extendido con la participación en los estudios y evaluaciones internacionales, como han sido el Proyecto de la Calidad de la Educación de la Oficina Regional de Educación para América Latina y el Caribe (OREALC), básicamente el SERCE, el TERCE y el ERCE correspondiente al presente año. ${ }^{4}$

Durante el año 2017 y 2018 se llevaron evaluaciones en el marco del Programa Internacional de Evaluación de Estudiantes para el Desarrollo (PISA-D) que se desarrolla en Cambodia, Ecuador, Guatemala, Honduras, Panamá, Paraguay, Senegal y Zambia. Los resultados en estas evaluaciones vuelven a mostrar el bajo rendimiento de los estudiantes guatemaltecos quienes se ubican por debajo de las medias de los demás países participantes. De la misma forma, develan que los principales factores asociados son relativos a las condiciones socioeconómicas, trabajo infantil, discriminación y exclusión, que exponen igualmente los estudios nacionales, Oficina Regional de Educación para América Latina y El Caribe, OREALC-UNESCO (2013).

En el presente año, 2019, el Consejo Nacional de Educación que está integrado por diversas representaciones de la sociedad guatemalteca, bajo la coordinación del Ministro de Educación, discutía cómo hacer efectiva la creación y funcionamiento del Sistema Nacional de Indicadores

\footnotetext{
${ }^{4}$ Segundo Estudio Regional Comparativo y Explicativo (SERCE, 2006); Tercer Estudio Regional Comparativo y Explicativo (TERCE, 2013); Estudio Regional Comparativo y Explicativo (ERCE, 2019).
} 
(SNIE), que fue creado mediante un acuerdo legal desde hace 6 años y, que hasta ahora, solo constituye parte del derecho vigente. El mencionado sistema combina un conjunto de propósitos heterogéneos, entre los cuales se menciona "el cumplimiento del mandato constitucional de garantizar a los guatemaltecos el goce pleno de su derecho a recibir una educación de calidad" (MINEDUC, 2013, p.1). Además de la búsqueda de la integración de los diferentes indicadores relativos a la cobertura educativa, rendimiento escolar y rendición de cuentas.

Los dos tipos de indicadores a los cuales se refiere el acuerdo son la cobertura y la evaluación del rendimiento escolar. Esto significa que se continúa bajo el mismo parámetro que lleva más de veinte años en el país. Pero el Sistema Nacional de Indicadores también es criticado en un contexto de tensiones que son legítimas en cuanto a la diversidad cultural del país. La falta de discusión y acuerdos con los pueblos indígenas condujo a la creación de otro Sistema de Indicadores que el actual Ministerio de Educación reguló mediante el Acuerdo Ministerial 493-2019, publicado el diario oficial en el presente año. Este acuerdo crea el Sistema de Indicadores técnico-pedagógicos de pertinencia cultural y lingüística e indicadores del cumplimiento de la educación bilingüe, como contrapartida al otro acuerdo y por considerar la falta de interés por los temas de los pueblos indígenas. No obstante, lo que se ha generado es una duplicidad de funciones y hasta cierto punto contradicciones por la creación de dos sistemas de indicadores, pero que ambos aún solo existen en papel.

El efecto mayor que se ha logrado durante los últimos años con las evaluaciones de rendimiento escolar en lectura y matemática en Guatemala, ha sido sin duda alguna las preocupaciones por resultados obtenidos en las pruebas a los graduandos. Pero estas preocupaciones se manifiestan fundamentalmente en la ciudad capital y en los estratos socioeconómicos más altos y medio altos (lo que representaría los quintiles $4^{\circ}$ y $5^{\circ}$ de la población). En otras palabras, los resultados de las evaluaciones de graduandos solo son los indicadores del $24 \%$ de jóvenes que tienen acceso a la educación y que, en su mayoría, estudian en los colegios privados $(70 \%$ del total $)$.

Por otra parte, uno de los consorcios de los medios de comunicación social del país que incluye radios, canales de televisión, prensa escrita y digital, con el apoyo de la DIGEDUCA, ha 
creado un ranking de colegios privados sobre la única base de los resultados de matemáticas y lectura, sin tomar en cuenta otros aspectos fundamentales en la formación integral de la persona. Los resultados se ofrecen en una categorización de logro y no logro, siendo catalogados como la máxima expresión de la calidad educativa en Guatemala. Esto quiere decir, que en la última década las evaluaciones han privilegiado a un grupo reducido de la población y creando clasificaciones insuficientes a costa de dejar de evaluar a la gente más pobre del país que en su mayoría solo asiste al nivel primario, perdiendo así la oportunidad de generar políticas efectivas que mejoren la calidad de la educación.

A pesar que el elemento principal de la calidad ha sido, durante los últimos años, la evaluación del rendimiento escolar de los graduandos, existen también otros criterios que son tomados en cuenta, tales como: la eficiencia interna del sistema educativo, particularmente los indicadores de número y tasas construidas a partir de la inscripción, permanencia, aprobación, culminación al quinto grado del nivel primario y otros. Finalmente, se ha incorporado la contabilización de los días efectivos de clase por parte de algunas organizaciones de la sociedad civil.

Uno de los avances en los procesos de evaluación ha sido la generación de mayores capacidades del personal de la DIGEDUCA. En la actualidad la mayoría de su equipo cuenta con una titulación hasta de nivel de maestría. Además, esta dependencia ha mantenido a su personal respetando su contratación permanente. En este sentido, el personal técnico ha ido acumulando aprendizajes que son fundamentales para el desarrollo de las evaluaciones. A pesar que el intercambio y la experiencia de participación en evaluaciones nacionales e internacionales y compartir con especialistas de otros países, ha contribuido a fortalecer el trabajo realizado, unos pocos técnicos han salido al exterior a realizar sus estudios de doctorado en investigación y evaluación, pero desafortunadamente al retornar no se han reincorporado a la institución.

La formación del personal técnico ha superado las dificultades de los años 90's del siglo XX cuando existió una sola maestría que brindaba la formación técnica y científica de la estructura que realizaba las evaluaciones. Esta maestría en evaluación e investigación educativa tenía 
un carácter latinoamericano con el apoyo de la Organización de Estados Americanos (OEA). La Universidad del Valle de Guatemala fue la responsable y contó con la participación de algunos profesores extranjeros del programa denominado Sistema de Medición de la Calidad de la Educación (SIMCE) de Chile; aunque esto tampoco significó la organización de una estructura similar a la del país sudamericano.

\section{Recomendaciones}

Para el análisis de la evaluación nacional de los rendimientos escolares en Guatemala se establecen algunos de sus principales desafíos y posibles vías de mejora:

a) Es necesaria una discusión que amplíe la comprensión y las acciones sobre la calidad educativa, tomando en consideración las diferencias entre pueblos y grupos de población. Los clamores de los pueblos indígenas no han sido tomados en cuenta como tampoco aspectos fundamentales de una población empobrecida. La relación entre una perspectiva bastante limitada de occidente ha dejado prácticamente de lado la pertinencia sociocultural.

La sociedad no puede concebirse como un todo cultural homogéneo. Pero desafortunadamente, nuestra mirada absurdamente ha sido dirigida hacia Europa o los Estados Unidos. De hecho, se establecieron un conjunto de estándares bajo el patrocinio de la USAID para los países del triángulo norte, El Salvador, Honduras y Guatemala. Esta medida no sólo provocó confusiones en los docentes, pero también desplegó una estrategia cuya base es la pretensión de reconducir los aprendizajes basados en competencias y concentrarse solo en enseñar para los exámenes en las escuelas. Sin embargo, esta dinámica ha tenido una repercusión mayor en los colegios privados que hoy concentran toda su atención en practicar los test para las evaluaciones.

Las evaluaciones, salvo los años comprendidos entre 1997 y 2000, no se han realizado en los idiomas mayas. En otras palabras, no existe ninguna preocupación de ampliar la dinámica evaluativa a otros mundos de vida. A este respecto, las publicaciones de la 
propia DIGEDUCA (2018) señalan que su participación en PISA-D tiene el propósito de asegurar que los objetivos educativos (en el caso de Guatemala representados por los estándares educativos y las competencias indicadas en el Currículo Nacional Base, $(\mathrm{CNB}))$ se encuentren alineados con el contenido de las evaluaciones.

En otras palabras, el currículo debe alinearse a las evaluaciones y no al revés. Más aún, en ningún momento se señala una línea clara respecto a las evaluaciones de la niñez de los pueblos indígenas. La reforma educativa producto de los Acuerdos de Paz que presupone el CNB, se funda en el reconocimiento de los pueblos indígenas y en la necesidad de llevar a cabo cambios profundos para resolver y construir una democracia intercultural, además de reconocer las diferencias en el marco de la equidad.

b) Debe de enfocarse la evaluación en el nivel primario y ciclo básico, que es donde asiste la mayoría de la población estudiantil de los diferentes estratos sociales y pueblos que conforman el país. Poner énfasis en los graduandos refleja la preocupación por un pequeño grupo que tiene al acceso a la educación. Más aun, como una narración aterradora, evaluar solo a los jóvenes graduandos se trata únicamente de medir el rendimiento de 22 de jóvenes de cada 100, puesto que el $78 \%$ no tiene acceso a este nivel educativo.

Además, que del total de este grupo la mayoría asiste al sector privado. Esto sin mencionar que los resultados más altos regularmente los obtienen los colegios con las cuotas más caras y que lejos de formar integralmente a los estudiantes, en realidad se preocupan cada vez más en las áreas del conocimiento evaluado. También preocupa que estos resultados tampoco obedecen a un proceso, un modelo de aprendizaje o modelo pedagógico. Es más bien producto del proceso de selección, en el cual los colegios van eliminando a los estudiantes que rinden mal.

c) El modelo de calidad educativa debe estar vinculado a políticas sociales y económicas que efectivamente mejoren las condiciones de las familias guatemaltecas. Analizando 
los resultados obtenidos por graduandos durante los últimos trece años, se puede observar que el peso de las variables estructurales es mayor que aquel que se refiere a factores controlados por las escuelas y sus comunidades. Esta situación también se corrobora con las evaluaciones de la UNESCO y de PISA-D. La pobreza, el trabajo infantil, la diversidad étnica y cultural afectan desfavorablemente en rendimiento escolar.

En este sentido, uno de los factores que más influye en los resultados de las pruebas es el nivel de educación de los padres. Los puntajes son significativamente mejores si cualquiera de los padres ha estudiado la secundaria alta. Por tanto, pareciera ser que el propio sistema mantiene y acrecienta las brechas sociales. De hecho, para el 2014 se calculaba que la pobreza de los adolescentes y jóvenes guatemaltecos era del $61.4 \%$ y solo el $38.6 \%$ era considerado como no pobre. El $24.1 \%$ fue considerado como pobre extremo y el $37.3 \%$ como pobre no extremo (Instituto Nacional de Estadística, INE, 2014).

d) Por otra parte, existe un olvido de cuestiones fundamentales como la formación ciudadana, la memoria histórica y el ámbito público de la educación. No digamos áreas relativas al reconocimiento de los saberes ancestrales mayas, las artes y las humanidades. La escuela guatemalteca en la práctica ha dejado de lado aquellos aprendizajes vinculados a la consecución del bien común, al aprendizaje de aquellas reglas y principios generales que apuntan al interés de la sociedad en equilibrio por supuesto del interés individual.

e) Equilibrar los balances entre lo que nuestro sistema considera como relevante y pertinente como parte de la calidad y equidad educativa. De lo contrario, se seguirá con un enfoque de evaluación que reduce la propia manifestación de la riqueza de la diversidad humana y natural, basándose exclusivamente a estándares cuyo resultado final será la profundización de las brechas entre grupos que afectan a las minorías étnicas y a los pobres o aquellos estudiantes que regularmente llamamos malos. 


\section{Consideración final}

En el fondo la educación y su evaluación requieren de una reforma más profunda, pues en la actualidad para el caso guatemalteco no trastoca las diferencias en la estructura social y las jerarquías entre los grupos, solo las expande. La educación, como parte de la intervención pública para atenuar los mecanismos y efectos de nuestro sistema económico y relaciones de poder, solo se manifiesta como un entramado que ahonda la desigualdad dejando de lado los principios de las sociedades democráticas. Esto quiere decir que, establecer parámetros de calidad sin considerar otras medidas esenciales para el desarrollo y la equidad, solo recrea condiciones que destruyen la posibilidad de la igualdad y la libertad.

\section{Referencias}

Chávez, J. (1989). Evaluación de logros en idioma español y matemática de los alumnos de sexto grado de primaria en Guatemala. Guatemala: Instituto de investigación y mejoramiento educativo- IME-USAC.

Comisión Centroamericana para la Reforma Educativa (2000). Mañana es muy tarde. Chile:

PREAL. Recuperado de http://1m1nttzpbhl3wbhhgahbu4ix.wpengine.netdna-cdn.com/ wp-content/uploads/2015/11/2000-Ma\%C3\%B1ana-es-Muy-Tarde-Comisi\%C3\%B3nCentroamericana-para-la-Reforma-Educativa.pdf

Dirección General de Evaluación e Investigación Educativa (2018). Guatemala en PISA-D. Recuperado de https://www.mineduc.gob.gt/digeduca/documents/pisa/ InformePISADGuatemala.pdf

Fortín, A. (2013). Evaluación Educativa Estandarizada en Guatemala: Un camino recorrido, un camino por recorrer. Guatemala: Ministerio de Educación.

Instituto Nacional de Estadística. (2014). Encuesta nacional de Condiciones de Vida. Guatemala: Gobierno de Guatemala.

Ministerio de Educación. (1988). Bases y elementos para la adecuación curricular. Guatemala: CENALTEX. 
Ministerio de Educación. (1999). Programa nacional de Evaluación del Rendimiento Escolar. Guatemala: Universidad del Valle de Guatemala.

Ministerio de Educación. (2008). Cultura de Evaluación. Boletín 1 Ajetab’al, p.3. Recuperado de http://www.mineduc.gob.gt/digeduca

Ministerio de Educación. (2010). La repitencia en primer grado, factores que influyen e impacto en los grados siguientes. Guatemala: Dirección General de Evaluación e Investigación Educativa.

Ministerio de Educación (2013). Acuerdo Ministerial 1703-2013 Creación del Sistema Nacional de Indicadores. Recuperado de http://www.mineduc.gob.gt/portal/ contenido/menu_lateral/leyes_y_acuerdos/leyes_educativas/documents/06_Acuerdos Varios_2013/1703-2013\%20Crear\%20Sistema\%20Nacional\%20de\%20Indicadores\%20 Educativos\%20-SNIE-.pdf

Ministerio de Educación. (2017). Informe de Factores Asociados al rendimiento de los estudiantes en la evaluación de primaria. Guatemala: Dirección General de Evaluación e Investigación Educativa.

Ministerio de Educación (2019). Acuerdo Ministerial 493-2019 Creación del Sistema de Indicadores Técnico-Pedagógicos de pertinencia cultural y lingüística. Recuperado de https://leyes.infile.com/index.php?id=182\&id_publicacion=79139

OREALC. (2013). Factores asociados al aprendizaje en el SERCE: análisis de los factores latentes y su vínculo con los resultados académicos de los niños. Oficina de Santiago: Laboratorio Latinoamericano de Evaluación de la Calidad de la Educación. 